FERMILAB-CONF-04-530-E

\title{
Tevatron Detector Upgrades
}

\author{
Ronald Lipton \\ Fermi National Accelerator Laboratory \\ Batavia, IL 60510, USA
}

\begin{abstract}
The D0 and CDF experiments are in the process of upgrading their detectors to cope with the high luminosities projected for the remainder of Tevatron Run II. We discuss the expected Tevatron environment through 2009, the detector challenges due to increasing luminosity in this period, and the solutions undertaken by the two experiments to mitigate detector problems and maximize physics results.
\end{abstract}

\section{HISTORY}

The Tevatron proton- antiproton collider has been operating since 1985 . Since that time the luminosity has increased by two orders of magnitude, the original CDF detector was joined by $\mathrm{D} 0$, and numerous improvements were made to the detectors and the accelerator complex. The original detectors used in the Top quark discovery were designed for luminosities of $\sim 10^{30} / \mathrm{cm}^{2}$ in a machine with six proton and antiproton bunches and collisions spaced by 3.5 microseconds.

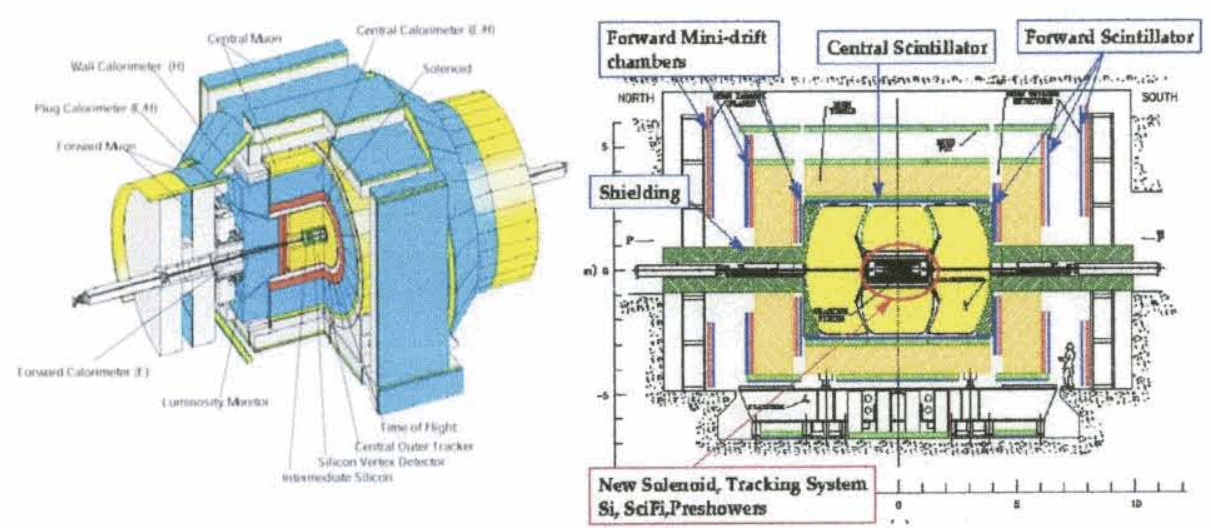

FIGURE 1. The CDF and D0 Detectors for Run II.

A major set of accelerator and detector upgrades preceded the start of Run II in 2001. On the accelerator side these included construction of the new Main Injector and Recycler rings, improvements to the antiproton source, and reduction of the Tevatron bunch spacing to $396 \mathrm{~ns}$. Both CDF and D0 undertook major upgrades to cope with the shorter bunch spacing and higher luminosity. Physics capabilities were 
improved by new tracking systems including extensive silicon detector systems in both experiments and the addition of a superconducting solenoid in D0.

As the Run II upgrades were proceeding the physics landscape was also changing. LEP and Tevatron measurements suggested a low value for the Higgs mass. A number of studies suggested that DO and CDF could discover the Higgs if its mass is below $150 \mathrm{GeV}$ as suggested by electroweak fits ${ }^{1}$ and the integrated luminosity delivered in Run II could be increased to $\sim 15 \mathrm{fb}^{-1}$. To achieve this goal before LHC turn-on required an increase in Tevatron peak luminosity to $\sim 5 \times 10^{32}$ and envisioned the use of the Recycler ring for antiproton storage and substantial improvements to the proton and antiproton source. The detectors and triggers would be further upgraded to cope with higher rates. Increased radiation damage to the silicon detectors was addressed by replacement of the Run IIa silicon by new radiation hard detectors. Problems due to occupancy would be mitigated by reducing the collision interval to $132 \mathrm{~ns}$ and implementing a luminosity leveling scheme. This was the original Run IIb upgrade program.

Experience in running the Tevatron Collider and the experiments have led to revisions in these plans. In the summer of 2003 a more conservative goal of $4-8 \mathrm{fb}^{-1}$ for the integrated luminosity in Run II was adopted. The complete replacement of the silicon detectors was cancelled in the Fall of 2003 and the upgrades were reduced to the more modest projects described in this talk.

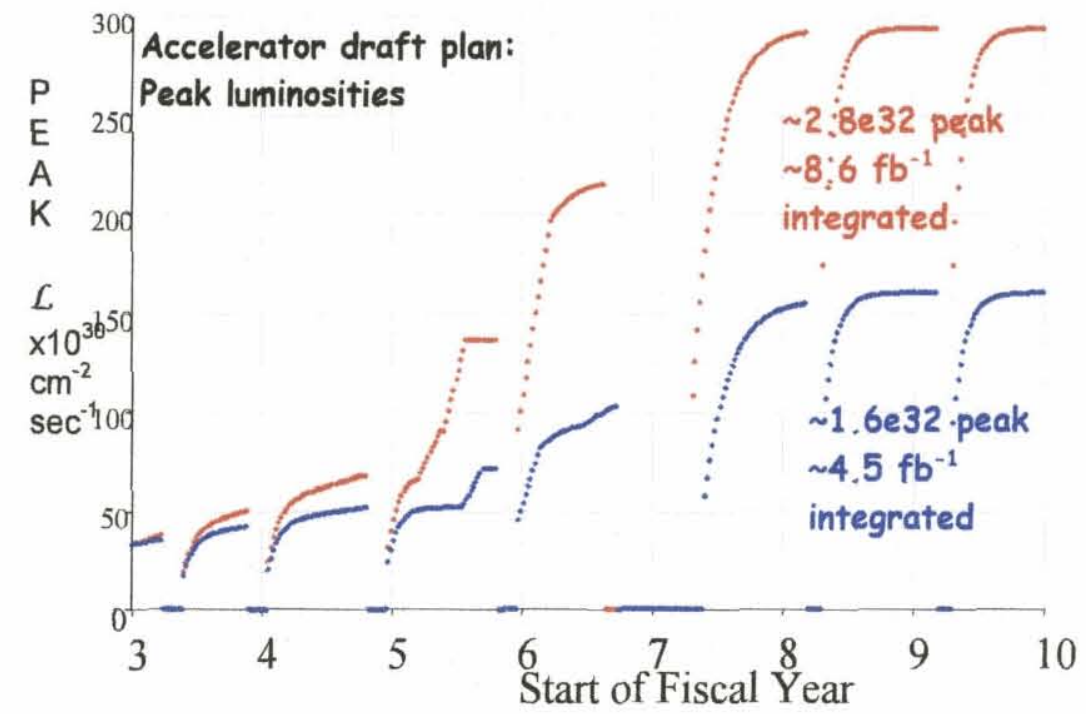

FIGURE 2. Draft plan for Tevatron luminosity through 2009 The upper (red) curves represent the "design" luminosity prediction, while the lower (blue) curves represent the "base" prediction.

\section{THE TEVATRON ENVIRONMENT}


The performance of the Tevatron has improved substantially in last six months, with increased store lengths, improved alignment, and improvements in the Tevatron lattice that have reduced $\beta^{*}$ at D0 from 43 to $34 \mathrm{~cm}$. Mixed mode stores utilizing antiprotons from the recycler ring have resulted in record luminosities. Given the recent success there is renewed confidence that the more aggressive "design" goals can be achieved. Full implementation of this plan requires the successful operation of electron cooling in the Recycler ring being installed in the Fall of 2004.

The Tevatron in Run II operates with 36 proton and antiproton bunches organized into three sets of 12-bunch trains. The luminous region typically has $\sigma_{x, y}=30 \mu \mathrm{m}, \sigma_{z}$ $=25 \mathrm{~cm}$. The crossing interval within the trains is $396 \mathrm{~ns}$, with 3 inter-train gaps of 2.6 $\mu \mathrm{s}$, one of which is used for beam abort kickers ${ }^{2}$. The predicted average number of interactions per crossing is 7 (2) for a 396 (132) ns crossing interval at $\mathrm{L}=2 \times 10^{32}$. Luminosity can vary among crossings by more than a factor of two due to uneven antiproton loading thus further increasing the instantaneous luminosity presented to the detectors. These multiple interactions at peak luminosity will pose a substantial challenge to trigger, DAQ and reconstruction. The 132 ns crossing interval option was abandoned in late 2002 based on concerns about beam dynamics and difficulties in implementing the many accelerator changes required. This makes the trigger upgrades, which focus on controlling rates due to multiple interactions, even more important. Some of the resulting experimental difficulties can be mitigated if luminosity leveling, shaping the luminosity profile by changing $\beta^{*}$ during the store while integrating comparable luminosity, can be implemented successfully. The Run IIb upgrades are intended to optimize detector performance for this environment.

\section{CDF AND D0 UPGRADES}

The Run II D0 and CDF detectors are shown in figure 1. The tracking and triggering systems are most relevant for the Run IIb upgrades. D0 tracking uses a barrel/disk Silicon Microstrip Tracker (SMT) surrounded by an eight layer scintillating fiber system within a 2 Tesla, 0.5 meter radius solenoid. CDF has an 8layer silicon tracker surrounded by a 96 layer drift chamber (COT) within a 1.4 Tesla solenoid with a radius of 1.5 meters.

Upgrades for Run IIb primarily address trigger and detector issues in the high luminosity environment ${ }^{3}{ }^{4}$. For CDF the upgrades replace gas chambers with scintillation detectors in the calorimeter and improve the time resolution for tracking triggers. For D0 there will be upgrades to the calorimeter and tracking triggers to improve energy and spatial resolution as well as the addition of a inner layer silicon detector.

\section{Trigger Upgrades}

Both experiments use a three level trigger system. The logical organization of the triggers (figure 3 ) are quite similar, with level 1 using information provided primarily by individual subsystems then combining the information at level 2 into a decision 
based on multiple systems. Level 3 farms than use the full precision detector information to make final decisions based on software event reconstruction

The rate capabilities of the two experiments are quite different (Table 1). CDF is "deadtimeless" at level 1, whereas D0 incurs deadtime due to the SVX II chips in the tracking system. The high level 1 bandwidth for CDF allows the use of sophisticated triggers at level 2, such as the SVT, to flag interesting events. With the existing $\sim 300$ $\mathrm{Hz}$ bandwidth limitation at level 2, the required rejection factor for CDF at level 2 is $\sim 200$. D0 has to filter effectively at level one, and depends on good muon purity and high quality level 1 calorimeter triggers to achieve an acceptable rate. Both experiments utilize fast tracking triggers at level 1 .

\begin{tabular}{|l|c|c|c|c|}
\hline \multicolumn{5}{|c|}{ Table 1. D0 and CDF trigger rate capabilities (Hz). } \\
\hline & D0 2004 & D0 Run IIb & CDF 2004 & CDF Run IIb \\
\hline Level 1 & 1500 & 2500 & 25000 & 25000 \\
\hline Level 2 & 850 & 1000 & 300 & 1000 \\
\hline Level 3 & 50 & $50-150$ & 50 & 85 \\
\hline
\end{tabular}

a) $\mathrm{D} 0$

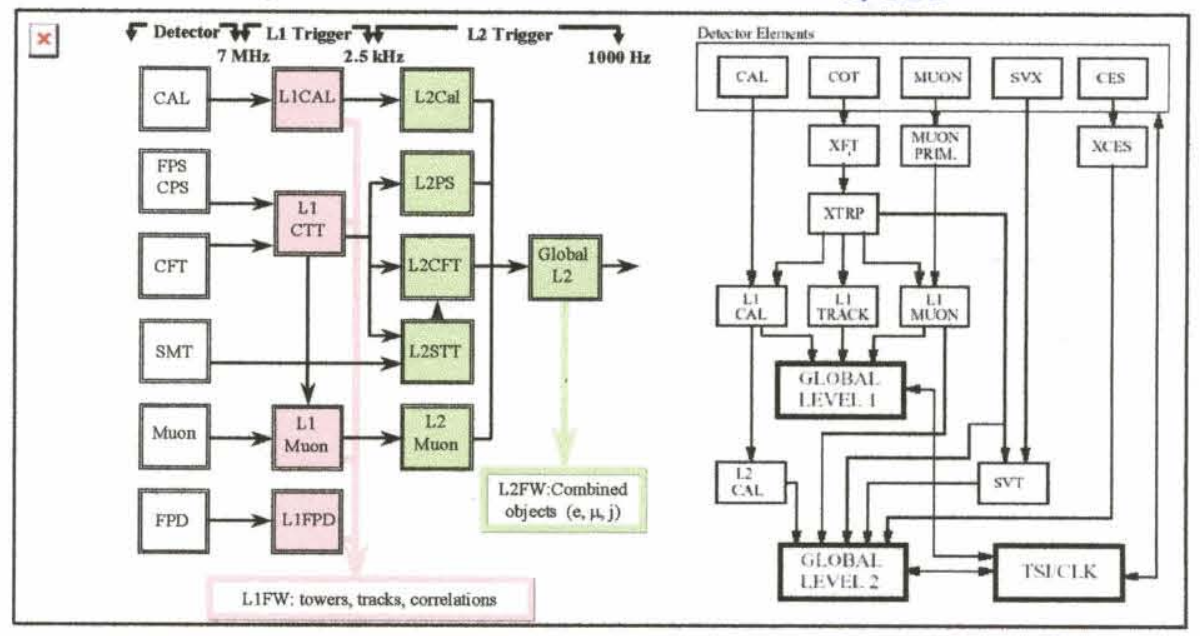

FIGURE 3. Block diagrams for the a) D0 and b) CDF level 1 and 2 triggers. In both cases level 3 uses the fully digitized data in a processor farm for the final trigger decision.

Both D0 and CDF tracking trigger upgrades are designed to improve the purity and efficiency of the tracking triggers by improving the effective resolution of the data presented to the trigger. For the CDF XFT (eXtremely Fast Tracker) this is achieved by adding stereo information to the XFT algorithm. New electronics will also allow faster readout to level 3, increasing level 2 throughput to $750 \mathrm{~Hz}$ from $300 \mathrm{~Hz}$, thus reducing the required level 2 rejection by a factor of 2.5. D0 will utilize larger FPGAs to implement a tracking algorithm using fiber singlets, rather than the doublet 
requirement currently implemented, which will have the effect of increasing tracking segmentation. The larger FPGAs also allow for the implementation of more flexible algorithms (eg. $\mathrm{N}$ of $\mathrm{M}$ layers rather than the current requirement for 8 hits in the 8 superlayers).

Both experiments plan to replace the vintage DEC Alpha processors used in level 2 with commodity Intel-based processors. The first phase of this is complete for D0. CDF also plans to replace its level 3 ATM switch with Gigabit Ethernet. A similar system is in place in D0.

D0 will install a new level 1 calorimeter trigger based on digital filtering of the liquid argon calorimeter signals. The digital filter will provide a sliding window for jet reconstruction. The sliding window substantially improves the turn-on edge for high Pt calorimeter triggers (figure 4). This allows D0 to keep high level 1 trigger efficiency for high Pt objects at acceptable level one trigger rates. The new calorimeter trigger will also allow matching of calorimeter energy to CTT tracks at level 1 .

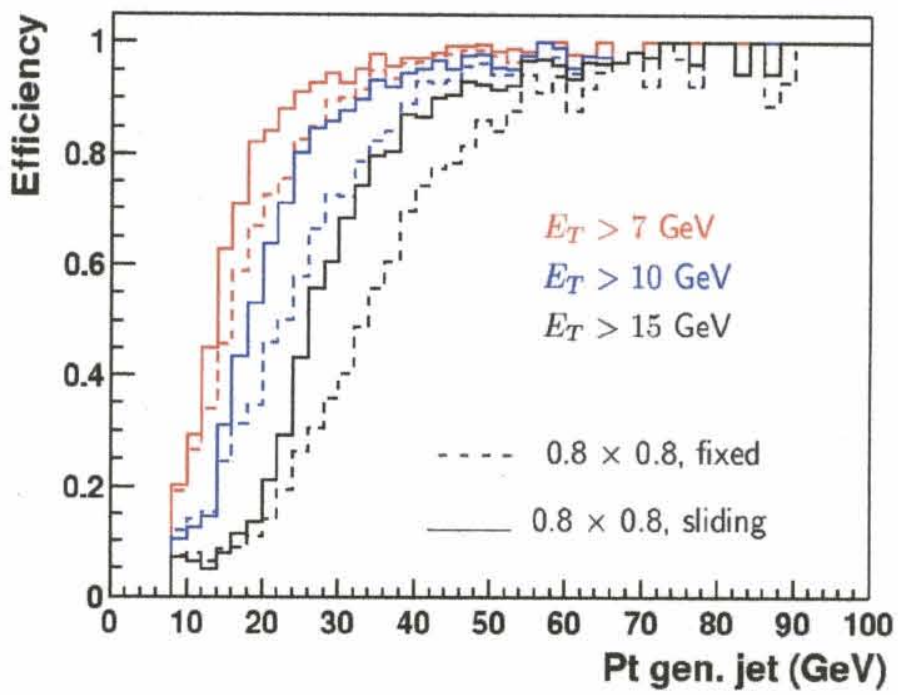

FIGURE 4. Trigger efficiency as a function of jet Pt for fixed and sliding windows algorithms.

\section{Detector Upgrades}

The CDF detector upgrades will complete the refurbishment of the CDF calorimeter system. The new Crack/Preshower detector (CPR) will tag energy in the calorimeter cracks and identify electromagnetic showers. These replace the existing gas detectors with scintillator/wavelength shifter modules based on technology developed for the plug calorimeter. Timing electronics will be added to the electromagnetic section of the calorimeter identical to the existing hadronic section 
hardware. This will give CDF the ability to accurately time electromagnetic showers and unambiguously identify cosmic ray backgrounds to rare events.

The cancellation of the silicon upgrades has left the collider experiments with silicon detectors which must survive irradiation 2-4 times their design dose. This is especially significant for D0, which has fewer silicon layers. In addition irradiation measurements on the double metal detectors used for the D0 inner layers have shown anomalously rapid reverse annealing which will result in a expected lifetime limited to $\sim 3.5 \mathrm{fb}^{-15}$.

To mitigate these losses, and improve the overall impact parameter resolution, D0 is building a "Layer 0" detector which is designed to be installed within the inner radius of the current SMT. This detector will consist of a single layer of radiation hard single-sided silicon detectors on a carbon fiber support structure. The Run IIb beam pipe will be used. Multiple scattering is minimized by utilizing analog cables to bring the signals to SVX4 hybrids placed outside of the active volume. Many of the technologies used for Layer 0 were developed for the original Run IIb silicon upgrades and use experience from the Run IIa silicon detectors, especially CDF Layer 00.
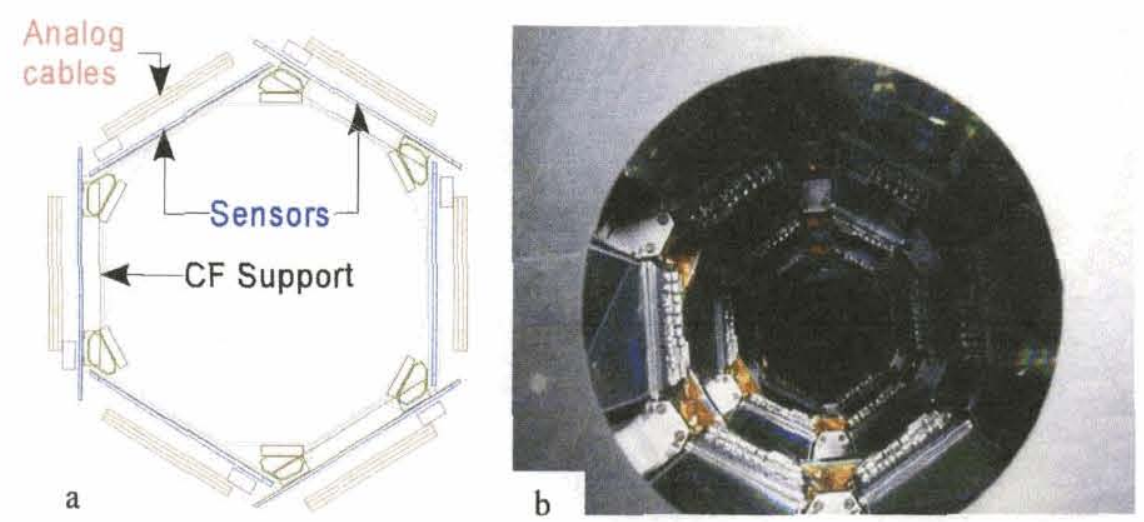

FIGURE 5. The DO Layer 0 detector a) cross section showing typical sensor locations and the analog cable stack at various positions b) photograph of the central hole in the Run IIa detector where Layer 0 will be inserted. Minimum clearance is expected to be $0.8 \mathrm{~mm}$.

\section{MAXIMIZING PHYSICS}

The addition of significant upgrades to a running experiment requires consideration of the balance between improvements in capability and time lost to installation and commissioning. The expected disruption to data taking due to the long installation period was the major reason given for cancellation of the Run IIb silicon detector upgrades. D0 upgrades to the calorimeter and fiber triggers also have the potential for significant disruption of the physics program. D0 has instituted a standing committee, 
which has the responsibility for understanding the work needed for installation and commissioning of the upgrades, and will advise the spokespersons as to whether the hardware, software, and infrastructure is ready for installation.

Both experiments have the ultimate goal of maximizing useful luminosity from the Tevatron in the pre-LHC era. This optimization includes the upgrades described in this talk, keeping high live fraction, and maintaining and understanding the existing detectors. For example CDF has successfully dealt with radiation damage to the inner layer of the COT by adding oxygen to the chamber gas, understood wirebond failures in the SVX, and solved cooling problems in the Intermediate Silicon Layer. D0 has solved problems with muon readout and is continuing to study calorimeter noise and to understand instabilities in SVXII chip readout in the silicon detector. Both experiments have steadily improved lifetime and tuned their trigger menus. These efforts complement the work being done to upgrade triggers and detectors given the expected increase in luminosity. Successful merging of upgrades with operations, continual tuning of hardware and software, and careful attention to data quality are essential components of successful running of the Tevatron experiments in the preLHC era.

\section{ACKNOWLEDGMENTS}

I would like to thank Pat Lukens and Morris Binkley for assistance with details of $\mathrm{CDF}$ upgrade plans and operations.

\section{REFERENCES}

1. "Report of the Run II Higgs Wotking Group",H M.Carena et al, hep-ph/0010338H.

2. "Run II Handbook", http://www-ad fnal.gov/runII/index.html.

3. Run IIb Upgrade Technical Design Report", FERMILAB-Publication-02/327-E (Dec 2002) 482p

4. "The Run IIb Technical Design Report", CDF Collaboration, CDF Note 6261.

5. "Lifetime of the DØ Silicon Tracker", D0 Collaboration, D0 note 4077. 